\title{
Order Reduction of the Dynamic Model of a Linear Weakly Periodic System-Part II: Frequency-Dependent Lines
}

\author{
Abner Ramirez, Adam Semlyen, and Reza Iravani
}

\begin{abstract}
The methodology for the order reduction of the dynamic model of a linear weakly periodic system presented in the first part of this paper is applied here to the case when the power network includes frequency dependent transmission lines. The technique for obtaining the external equivalent of the network is described. A comparison is given between systems having lines with constant or frequency-dependent parameters. Time-domain simulations have shown that the reduced-order equivalent leads to fast and accurate results.
\end{abstract}

Index Terms-Harmonic power flow, state-space methods, timeperiodic systems, time-varying systems, traveling waves.

\section{INTRODUCTION}

HE THEORY for reducing the dynamic model of a linear weakly periodic (LWP) system has been presented in the first part of this paper [1]. As described there, the basis for the reduction is the assumption of the system being LWP in steady state ("weakly" means that its linear part is dominant) so that the system can be linearized around its periodic state. Initially, the order of the reduced system is defined by the reduction of its linear part. Then, the time-periodic harmonic part for the reduced system can be obtained via a general method based on Gauss-Seidel iterations, or by a simplified approach based on a forced extremely sparse solution. It is shown in [1] that the latter leads to results of the same accuracy as the general procedure but with higher computational efficiency.

In this second part of the paper, we present an approach for the inclusion of frequency-dependent (FD) lines in the network. The transmission line is represented in the form of a state-space realization obtained by rational approximation of its admittance matrix [2]. This model is hereafter referred to as admittance-frequency-dependent approach (Y-FD). On the other hand, the model used in [1] is referred to as a constant parameters approach (CP).

Consideration of FD line modeling permits us to obtain more accurate results in the time-domain simulations. However, the proposed technique can also be applied to other electrical devices for which taking frequency dependence into account is important. Among the potential applications of the proposed

Manuscript received August 5, 2003. The work of A. Ramirez was supported by the Natural Sciences and Engineering Research Council of Canada (NSERC) and by the Science and Technology Council of Mexico (CONACYT).

The authors are with the Department of Electrical and Computer Engineering, University of Toronto, Toronto, ON M5S3G4, Canada (e-mail: abner@power.ele.utoronto.ca; adam.semlyen@utoronto.ca; iravani@ecf. utoronto.ca).

Digital Object Identifier 10.1109/TPWRS.2003.821619 methodology are situations when there is a need to use detailed models of transformers or of switching elements; when the corona effect is present in the lines; and also the study of harmonic resonances in an external system and detection of dominant poles [3].

For added convenience, clarity, and emphasis, we first present in Section II a brief review of the methodology described in [1]. Then, Section III describes the modeling of an external system to be reduced including FD lines. Section IV gives an example and the corresponding results.

\section{BRIEF REVIEW OF THE BASIC THEORY}

This section reviews the simplified alternative of the general order-reduction methodology described in [1]. The main objective of [1] is to describe a procedure for transforming the linearized (initially nonlinear, time-varying) system of order $N$ (the incremental notation is omitted)

$$
\begin{aligned}
& \dot{x}=A x+B u \\
& y=C x
\end{aligned}
$$

into an equivalent system of a reduced order $r$

$$
\begin{aligned}
& \dot{z}=E z+F u \\
& y=G z
\end{aligned}
$$

via the transformation

$$
z=S x
$$

The systems (1) and (2) contain $T$-periodic matrices ( $A$ to $G$ ) having the following typical structure:

$$
A=A_{o}+\sum_{h=1}^{H_{\max }}\left(A_{b} e^{j h \omega_{o} t}+A_{-h} e^{-j h \omega_{o} t}\right)
$$

where $\omega_{o}$ represents the fundamental angular frequency and $H_{\text {max }}$ denotes the highest harmonic considered in the transient study. As the original system is assumed to be LWP, first the constant part corresponding to $E_{o}, F_{O}$, and $G_{O}$ is calculated via a reduction based on singular value decomposition (SVD). Then the harmonic coefficient matrices $E_{h}, F_{h}$, and $G_{h}$ are obtained using the transformation matrix $S$. This matrix can be considered as a $T$-periodic matrix or as a constant matrix, $S_{o}$. The first option leads to a Gauss-Seidel procedure. The second option, used in this paper, requires no iterative procedures, is more efficient computationally, and preserves the accuracy of 
the reduction. The relations for this simplified approach are ( $G_{h}$ is assumed to be zero)

$$
\begin{aligned}
z & =S_{o} x \\
E_{h} & =S_{o} A_{h} S_{o}^{T} \\
F_{h} & =S_{o} B_{h} .
\end{aligned}
$$

From (5b), $E_{h}$ is obtained as a subset of $A_{h}$ by the relation $E_{h}(i, j)=A_{h}(k, l)$, with $i, j=1, \ldots n$ and $k, l=$ vector of positions for the columns of $S_{o}$ containing "ones" [1].

\section{NETWORK WiTH FD TRANSMISSION LINES}

In this section, we present the assemblage of state equations for a FD transmission line (or a network) with nonlinear load(s).

\section{A. Admittance Approach ( $Y-F D)$}

1) Single Transmission Line: The two-port voltage/current relation for the transmission line depicted in Fig. 1 can be expressed as

$$
\left[\begin{array}{c}
I_{m} \\
I_{n}
\end{array}\right]=\left[\begin{array}{ll}
Y_{1} & Y_{2} \\
Y_{2} & Y_{1}
\end{array}\right]\left[\begin{array}{l}
V_{m} \\
V_{n}
\end{array}\right]
$$

where

$$
\begin{aligned}
& Y_{1}=Y_{c} \operatorname{coth}(\gamma l) \\
& Y_{2}=-Y_{c} \operatorname{csch}(\gamma l) .
\end{aligned}
$$

In (7a) and (7b), $Y_{c}$ and $\gamma$ represent the characteristic admittance and the propagation function, respectively, of a line of length $l$. Approximating $Y_{1}$ and $Y_{2}$ from (7) by rational functions [4], we can express the first equation in (6) as follows:

$I_{m}=\left(C_{1}\left(s I-A_{1}\right)^{-1} B_{1}+D_{1}\right) V_{m}+C_{2}\left(s I-A_{1}\right)^{-1} B_{1} V_{n}$.

Note that the same set of poles, contained in the diagonal matrix $A_{1}$, is used for both admittances. In addition, the same column vector $B_{1}$, with all entries equal to 1 in the single phase case, is used for both admittances. The residues of the realization are contained in $C_{1}$ and $C_{2}$. From (8), we define

$$
\begin{aligned}
& X_{1}=\left(s I-A_{1}\right)^{-1} B_{1} V_{m} \\
& X_{2}=\left(s I-A_{1}\right)^{-1} B_{1} V_{n} .
\end{aligned}
$$

In the time domain, (8) and (9) become

$$
\begin{aligned}
{\left[\begin{array}{c}
\dot{x_{1}} \\
\dot{x_{2}}
\end{array}\right] } & =\left[\begin{array}{ll}
A_{1} & \\
& A_{1}
\end{array}\right]\left[\begin{array}{l}
x_{1} \\
x_{2}
\end{array}\right]+\left[\begin{array}{ll}
B_{1} & \\
& B_{1}
\end{array}\right]\left[\begin{array}{l}
v_{m} \\
v_{n}
\end{array}\right], \\
i_{m} & =\left[\begin{array}{ll}
C_{1} & C_{2}
\end{array}\right]\left[\begin{array}{l}
x_{1} \\
x_{2}
\end{array}\right]+D_{1} v_{m} .
\end{aligned}
$$

The definitions in (9) and the (10a) apply also for node $n$ of the line. Thus, we have the following complete state-space realization for the transmission line:

$$
\begin{aligned}
& {\left[\begin{array}{c}
\dot{x_{1}} \\
\dot{x_{2}}
\end{array}\right]=\left[\begin{array}{ll}
A_{1} & \\
& A_{1}
\end{array}\right]\left[\begin{array}{l}
x_{1} \\
x_{2}
\end{array}\right]+\left[\begin{array}{ll}
B_{1} & \\
& B_{1}
\end{array}\right]\left[\begin{array}{l}
v_{m} \\
v_{n}
\end{array}\right]} \\
& {\left[\begin{array}{c}
i_{m} \\
i_{n}
\end{array}\right]=\left[\begin{array}{ll}
C_{1} & C_{2} \\
C_{2} & C_{1}
\end{array}\right]\left[\begin{array}{l}
x_{1} \\
x_{2}
\end{array}\right]+D_{1}\left[\begin{array}{l}
v_{m} \\
v_{n}
\end{array}\right] .}
\end{aligned}
$$

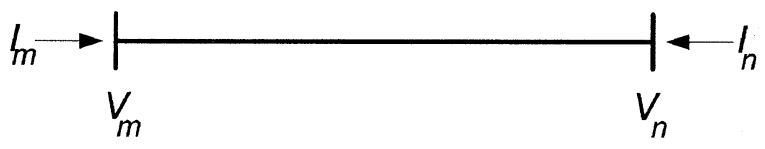

Fig. 1. Reference directions, single transmission line.

Consider now that a nonlinear load, consisting of a saturating reactor, is connected at node $n$ and is described by

$$
\begin{aligned}
i_{\text {load }} & =\alpha \varphi+\beta \varphi^{3} \\
\dot{\varphi} & =v_{n} .
\end{aligned}
$$

If we apply KCL at node $n, i_{n}+i_{\text {load }}=0$, then the voltage at this node can be calculated from (11b) and (12a) as a function of the state variables from

$$
v_{n}=-\frac{\left(C_{2} x_{1}+C_{1} x_{2}+\alpha \varphi+\beta \varphi^{3}\right)}{D_{1}} .
$$

Then, (13) is substituted into (11) and (12b) to obtain the final expressions

$$
\begin{aligned}
{\left[\begin{array}{c}
\dot{x}_{1} \\
\dot{x}_{2} \\
\dot{\varphi}
\end{array}\right]=} & \frac{1}{D_{1}}\left[\begin{array}{ccc}
A_{1} D_{1} & 0 & 0 \\
-B_{1} C_{2} & A_{1} D_{1}-B_{1} C_{1} & -B_{1}\left(\alpha+\beta \varphi^{2}\right) \\
-C_{2} & -C_{1} & -\left(\alpha+\beta \varphi^{2}\right)
\end{array}\right] \\
& \times\left[\begin{array}{c}
x_{1} \\
x_{2} \\
\varphi
\end{array}\right]+\left[\begin{array}{c}
B_{1} \\
0 \\
0
\end{array}\right] v_{m} \\
i_{m}= & {\left[\begin{array}{lll}
C_{1} & C_{2} & 0
\end{array}\right]\left[\begin{array}{c}
x_{1} \\
x_{2} \\
\varphi
\end{array}\right]+D_{1} v_{m} . }
\end{aligned}
$$

The equations in (14) define the input-output relation in the time domain for the transmission line viewed from node $m$. The effect of the nonlinear load has been included analytically by elimination of the currents at node $n$ of the line. Assuming the system in steady state, the initial conditions for (14) are obtained from its harmonic domain (HD) representation [5].

2) Network of Transmission Lines: In the case of a network with several transmission lines, the "node elimination" technique described above for one line is still applicable for obtaining its external equivalent. For illustration, this technique is briefly described in the following for the system from Fig. 2 (see Appendix A for details) but it can be used for any general network as well.

First, relations similar to (11a) and (11b) are obtained for each line. Then, KCL is applied to each node to calculate the corresponding voltage as a function of the local and neighboring states. These voltages are then substituted into the dynamic equations similar to (11a) for each line to obtain the final input-output relation as in (14). This procedure yields a complete system of the form

$$
\begin{aligned}
{\left[\begin{array}{c}
\dot{x} \\
\dot{\varphi}
\end{array}\right] } & =\left[\begin{array}{ll}
A_{\text {const }} & A_{\text {nonlin }}
\end{array}\right]\left[\begin{array}{l}
x \\
\varphi
\end{array}\right]+B v_{1} \\
i_{1} & =C\left[\begin{array}{l}
x \\
\varphi
\end{array}\right]+D_{1} v_{1}
\end{aligned}
$$

where $x$ and $\varphi$ contain the states from the lines and loads, respectively. The dynamic matrix is formed mainly by a con- 
stant part $A_{\text {const }}$, corresponding to a combination of poles and residues from the lines, and a time-varying part $A_{\text {nonlin, }}$, corresponding to the nonlinear loads.

The state equations in (15) correspond to a system of nonlinear equations. For the purpose of reduction, this system has to be linearized around the periodic steady state as shown in [1] to obtain the complete linearized system as in (1). The improvement in this second part of the paper consists of the inclusion of a more accurate model for the transmission lines via the approximation of their admittance matrices by rational functions.

\section{B. Traveling Wave Approach (TW-FD)}

In the admittance approach described above, the functions in (7) are approximated by rational functions. This approximation requires a large number of poles due to many peaks involved in the hyperbolic functions. Thus, (15) represents ODEs containing a large number of states which is transformed into ODEs of reduced order. A more common approach, based on traveling waves, is to represent the transmission line by the characteristic admittance and the propagation function as its fundamental parameters [6]. This is referred to hereafter as traveling-wave frequency-dependent approach (TW-FD). These parameters are smoother functions than those from (7) and can be approximated by a lower order ODE realization. However, in this approach the representation equivalent to (15), corresponding to traveling waves, is delay differential equations (DDEs) (see, for example, [7]) for which there is no available order-reduction technique. In this paper, the complete DDEs are used to simulate the original system for comparison with its counterpart in (15). Details of the construction of the DDEs are given in Appendix B. The order reduction of a DDE system could be the object of interesting future research, as suggested in Fig. 3.

\section{EXAMPLE}

The configuration of the network used for this example is presented in Appendix C. The results for the three approaches used, namely admittance (Y-FD), constant parameters (CP), and traveling wave (TW-FD), are presented in this section.

A harmonic power flow (HPF) program is utilized to obtain the steady state of the network using up to the ninth harmonic. Note that an HPF capable of handling FD lines is used for the admittance approach. The line constants in the $\mathrm{CP}$ approach are calculated at the frequency of $100 \mathrm{~Hz}$ to match the steady-state and the harmonic content corresponding to the FD approaches.

The nonlinear characteristic (12a) of the load at bus 2 is presented in Fig. 4 for illustration. The per-unit flux in Fig. 4 is calculated with the base voltage of $15 \mathrm{kV}$.

In the Y-FD approach, each line is fitted up to $160 \mathrm{kHz}$ with 84 poles (40 complex pairs and 4 real) resulting in a total of $N=2535$ states, which are reduced to $r=54$. The HPF yields the following ratios of harmonic content in the output current: $I_{3} / I_{\mathrm{o}}=0.10, I_{5} / I_{\mathrm{o}}=0.0075, I_{7} / I_{\mathrm{o}}=0.0015$, and $I_{9} / I_{\mathrm{O}}=0.0001$.

In the case of the CP approach (discussed in [1]), each line is approximated by $84 \pi$-sections, resulting in a total of $N=2537$ states which are reduced to $r=57$. The HPF yields the fol-

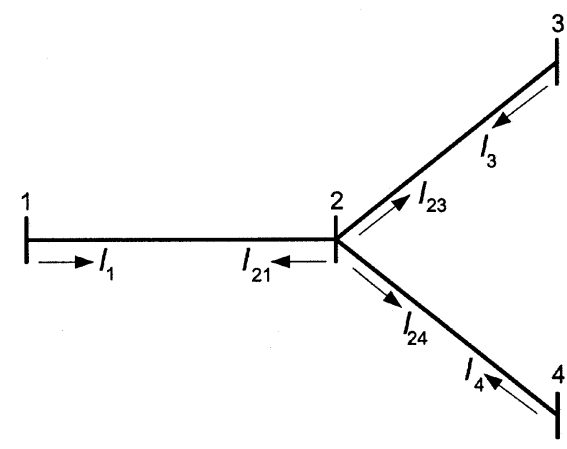

Fig. 2. Reference directions, three transmission lines.

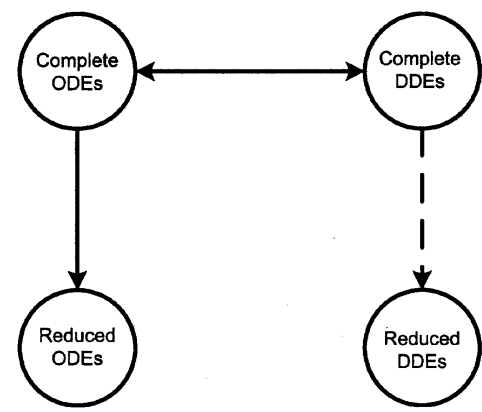

Fig. 3. Two different approaches for order reduction.

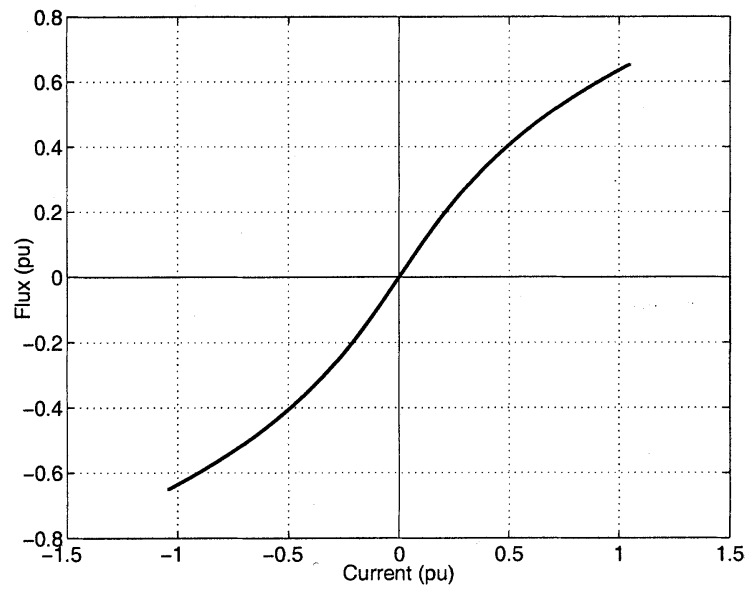

Fig. 4. Current-flux characteristic at bus 2 for the example.

lowing ratios of harmonic content in the output current $I_{3} / I_{\mathrm{O}}=$ $0.096, I_{5} / I_{\mathrm{o}}=0.0072, I_{7} / I_{\mathrm{o}}=0.0017$, and $I_{9} / I_{\mathrm{o}}=0.00006$.

Fig. 5 shows the simulation of the original nonlinear and reduced systems using both, the Y-FD and CP approaches. From Fig. 5, we can observe that in the Y-FD approach the high frequencies are effectively attenuated while with the use of the $\mathrm{CP}$ model they are not, leading to inaccurate results. Note from the dotted lines that the order reduction has been obtained in a highly effective manner in both approaches (see also Table I).

The same network was also simulated using the TW-FD approach. In this case, each line is fitted with eight real poles for both the characteristic admittance and the propagation function. The comparison between the TW-FD and the Y-FD approaches is shown in Fig. 6. In addition, the steady-state solution from 


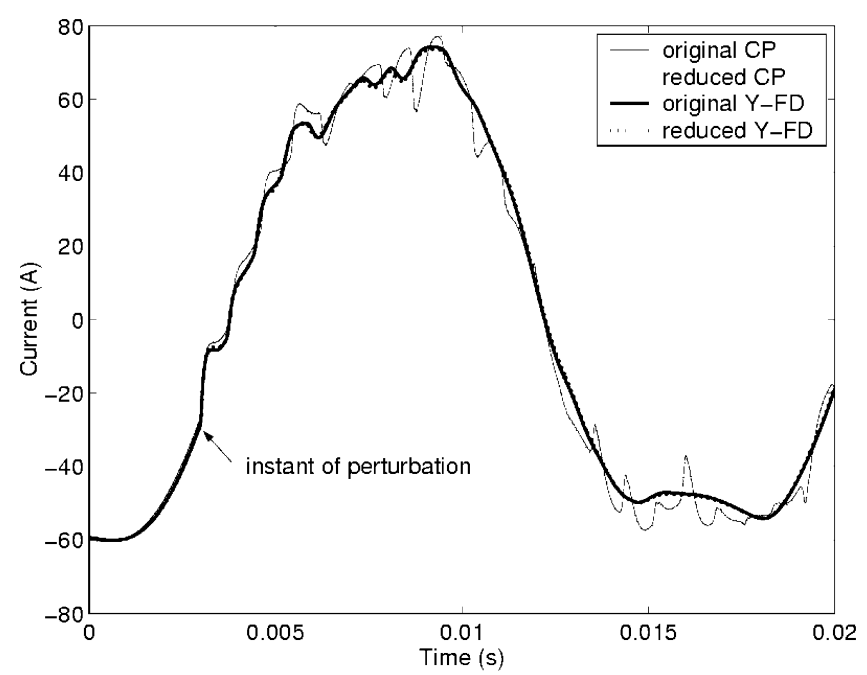

(a)

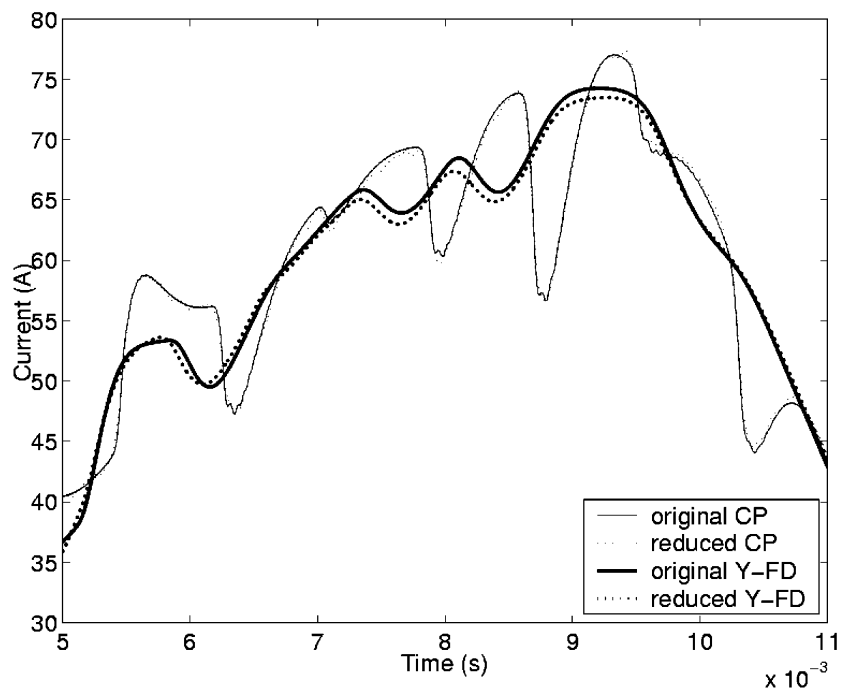

(b)

Fig. 5. (a) Original and reduced systems, considering frequency dependence of the line parameters (Y-FD) and with constant parameters (CP). (b) Close-up.

TABLE I

TIME AND RELATIVE ERRORS

\begin{tabular}{c|c|c|c|c|c}
\hline \multirow{2}{*}{ MODEL } & \multicolumn{2}{|c|}{ Y-FD } & \multicolumn{2}{c|}{ CP } & TW-FD \\
\cline { 2 - 6 } & Error \% & Time & Error \% & Time & Time \\
\hline Original & 0.0 & $147 \mathrm{~min}$ & 0.0 & $117 \mathrm{~s}$ & $64 \mathrm{~min}$ \\
\hline Reduced & 1.2 & $138 \mathrm{~s}$ & 0.8 & $159 \mathrm{~s}$ & - \\
\hline
\end{tabular}

the HPF is also presented in Fig. 6. In theory, these approaches are equivalent and this is confirmed in the figure. We note that, as expected with FD line representation, the higher frequency components in the transient attenuate faster than the lower frequencies. In Fig. 6, we see that the initial effect of the perturbation is first amplified and a significant disturbance persists for a long time. This is likely due to resonance effects in the system.

The relative errors (as a percentage, with respect to the original) and the computational time for the example are presented in Table I. The computer used for obtaining all results in this paper is a Pentium III, 1-GHz speed, 512-MB RAM.

Table I shows that if the transmission line is approximated by $\pi$-sections (CP), the time domain simulation becomes much

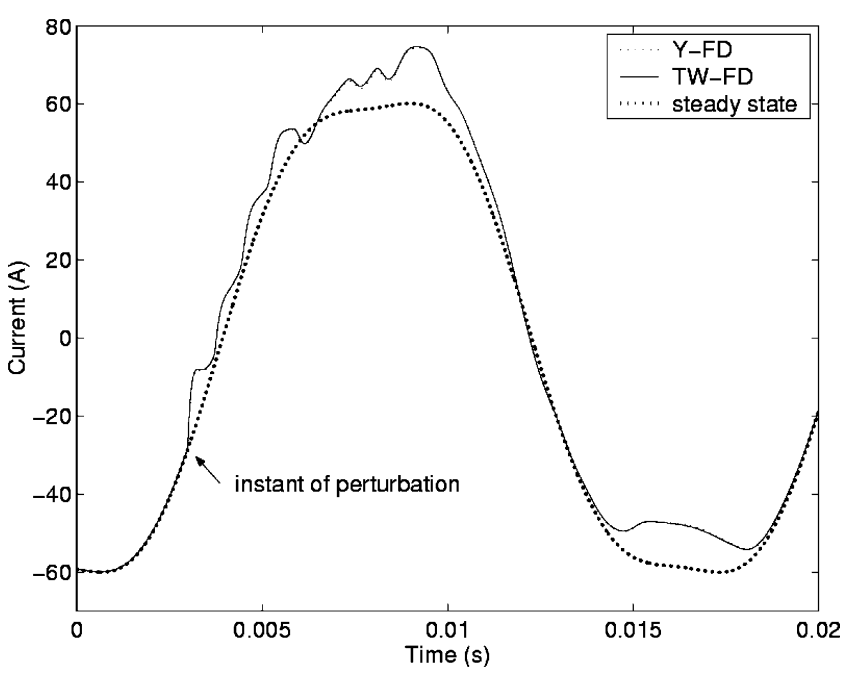

Fig. 6. Comparison between original Y-FD and original TW-FD approaches, and steady state from HPF.

faster than in the case of FD line representation. However, the latter provides more accurate results. Table I also shows that, in the admittance approach, the simulation time for the reduced order system is about $1.5 \%$ of that corresponding to the original system. It also indicates that the simulation time corresponding to the original TW-FD approach is less than that of the original Y-FD approach. This is an expected result due to the smaller number of poles involved in the TW-FD approach. The computation times in Table I correspond to Matlab implementation and these could be significantly reduced if, for example, Fortran-based programs are used.

\section{CONCLUSIONS}

The modeling of a FD transmission line has been shown in this part of the paper for the order-reduction methodology presented in [1]. This more sophisticated line model has permitted to obtain more accurate simulation of the phenomenon when the LWP system is perturbed. The results presented here have demonstrated the accuracy of the reduction method and the computational efficiency achieved by the reduced order equivalent. The example presented contains only transmission lines and nonlinear loads but the proposed reduction technique is suitable for modeling other complex components such as transformers and switching elements.

\section{APPENDIX A}

In this section, the nodal elimination technique is described for the system presented in Fig. 2. First, for each line the voltage/current relation as in (6) can be expressed in the form of state equations similar to (11)

$$
\begin{aligned}
& {\left[\begin{array}{l}
\dot{x}_{1} \\
\dot{x}_{2}
\end{array}\right]=\left[\begin{array}{ll}
A_{1} & \\
& A_{1}
\end{array}\right]\left[\begin{array}{l}
x_{1} \\
x_{2}
\end{array}\right]+\left[\begin{array}{ll}
B_{1} & \\
& B_{1}
\end{array}\right]\left[\begin{array}{l}
v_{1} \\
v_{2}
\end{array}\right]} \\
& {\left[\begin{array}{c}
i_{1} \\
i_{21}
\end{array}\right]=\left[\begin{array}{ll}
C_{1,1} & C_{2,1} \\
C_{2,1} & C_{1,1}
\end{array}\right]\left[\begin{array}{l}
x_{1} \\
x_{2}
\end{array}\right]+D_{1}\left[\begin{array}{l}
v_{1} \\
v_{2}
\end{array}\right]} \\
& {\left[\begin{array}{l}
\dot{x}_{3} \\
\dot{x}_{4}
\end{array}\right]=\left[\begin{array}{ll}
A_{2} & \\
& A_{2}
\end{array}\right]\left[\begin{array}{l}
x_{3} \\
x_{4}
\end{array}\right]+\left[\begin{array}{ll}
B_{2} & \\
& B_{2}
\end{array}\right]\left[\begin{array}{l}
v_{2} \\
v_{3}
\end{array}\right]}
\end{aligned}
$$




$$
\begin{aligned}
& {\left[\begin{array}{c}
i_{23} \\
i_{3}
\end{array}\right]=\left[\begin{array}{ll}
C_{1,2} & C_{2,2} \\
C_{2,2} & C_{1,2}
\end{array}\right]\left[\begin{array}{l}
x_{3} \\
x_{4}
\end{array}\right]+D_{2}\left[\begin{array}{l}
v_{2} \\
v_{3}
\end{array}\right]} \\
& {\left[\begin{array}{l}
\dot{x}_{5} \\
\dot{x}_{6}
\end{array}\right]=\left[\begin{array}{ll}
A_{3} & \\
& A_{3}
\end{array}\right]\left[\begin{array}{l}
x_{5} \\
x_{6}
\end{array}\right]+\left[\begin{array}{ll}
B_{3} & \\
& B_{3}
\end{array}\right]\left[\begin{array}{l}
v_{2} \\
v_{4}
\end{array}\right]} \\
& {\left[\begin{array}{c}
i_{24} \\
i_{4}
\end{array}\right]=\left[\begin{array}{ll}
C_{1,3} & C_{2,3} \\
C_{2,3} & C_{1,3}
\end{array}\right]\left[\begin{array}{l}
x_{5} \\
x_{6}
\end{array}\right]+D_{3}\left[\begin{array}{l}
v_{2} \\
v_{4}
\end{array}\right] .}
\end{aligned}
$$

Then, KCL is applied to each node to calculate the corresponding voltage as a function of the local and neighboring states. For example, at node 2 the relation $i_{21}+i_{23}+i_{24}+$ $i_{\text {load2 }}=0$ is applied to obtain the voltage $v_{2}$ is as shown in the equation at the bottom of the page. At node 3 , we have that $i_{3}+i_{\text {load } 3}=0$, thus $v_{3}$ is given by

$$
v_{3}=-\frac{\left(C_{2,2} x_{3}+C_{1,2} x_{4}+\alpha_{2} \varphi_{2}+\beta_{2} \varphi_{2}^{3}\right)}{D_{2}} .
$$

Similarly, at node 4 , the relation $i_{4}+i_{\text {load } 4}=0$ leads to

$$
v_{4}=-\frac{\left(C_{2,3} x_{5}+C_{1,3} x_{6}+\alpha_{3} \varphi_{3}+\beta_{3} \varphi_{3}^{3}\right)}{D_{3}} .
$$

The voltages from (19) are then substituted into (16a), (17a), and (18a) to obtain the final relations (15). In (15a), the column vector $B$ contains as entries only the elements from $B_{1}$ and the output current in (15b), $i_{1}$, is extracted from the first equation of (16b).

\section{APPENDIX B \\ TW-FD}

\section{A. Single Transmission Line}

Consider the transmission line shown in Fig. 7 with a nonlinear load, represented by (12), connected at end $n$. The relations between the incident current $I^{\prime}$ and the reflected current $I^{\prime \prime}$ are

$$
\begin{aligned}
I_{m}^{\prime} & =H I_{n}^{\prime \prime} \\
I_{n}^{\prime} & =H I_{m}^{\prime \prime}
\end{aligned}
$$

where $H$ represents the propagation function. The state-space realization for (20a) and (20b) is

$$
\begin{aligned}
& {\left[\begin{array}{l}
\dot{x}_{1} \\
\dot{x}_{2}
\end{array}\right]=\left[\begin{array}{ll}
A_{1} & \\
& A_{1}
\end{array}\right]\left[\begin{array}{l}
x_{1} \\
x_{2}
\end{array}\right]+\left[\begin{array}{ll}
B_{1} & \\
& B_{1}
\end{array}\right]\left[\begin{array}{l}
i_{n}^{\prime \prime} \\
i_{m}^{\prime \prime}
\end{array}\right]} \\
& {\left[\begin{array}{l}
i_{m}^{\prime} \\
i_{n}^{\prime}
\end{array}\right]=\left[\begin{array}{ll}
C_{1} & \\
& C_{1}
\end{array}\right]\left[\begin{array}{l}
x_{1} \\
x_{2}
\end{array}\right]}
\end{aligned}
$$

where $A_{1}, B_{1}$, and $C_{1}$ have the same structure as for the admittance approach. In (21), the reflected currents $i_{n}^{\prime \prime}$ and $i_{m}^{\prime \prime}$ are calculated at time $t-\tau, \tau$ being the travel time. In addition, we have the relations

$$
\begin{aligned}
Y_{c} V_{m}-I_{m} & =2 I_{m}^{\prime} \\
Y_{c} V_{n}-I_{n} & =2 I_{n}^{\prime} .
\end{aligned}
$$

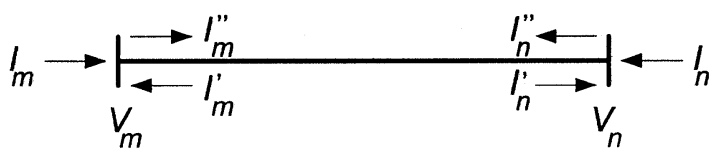

Fig. 7. Reference directions, single transmission line.

Assuming that the voltage at node $m$ is known, the realization for (22a) is

$$
\begin{aligned}
& \dot{x}_{3}=A_{2} x_{3}+B_{2} v_{m} \\
& i_{m}=C_{2} x_{3}+D_{1} v_{m}-2 i_{m}^{\prime} .
\end{aligned}
$$

The reflected current is then updated with

$$
i_{m}^{\prime \prime}=i_{m}^{\prime}+i_{m}
$$

Similarly, from (22b) for node $n$, we have

$$
\begin{aligned}
\dot{x}_{4} & =A_{2} x_{4}+B_{2} v_{n} \\
i_{n} & =C_{2} x_{4}+D_{1} v_{n}-2 i_{n}^{\prime} .
\end{aligned}
$$

Considering a nonlinear load as in (12) for node $n$, the voltage $v_{n}$ can be eliminated from (25) by application of KCL. Thus, the realization for this node including the nonlinear load is

$$
\begin{aligned}
{\left[\begin{array}{c}
\dot{x}_{4} \\
\dot{\varphi}
\end{array}\right]=} & \frac{1}{D_{1}}\left[\begin{array}{cc}
A_{2} D_{1}-B_{2} C_{2} & -B_{2}\left(\alpha+\beta \varphi^{2}\right) \\
-C_{2} & -\left(\alpha+\beta \varphi^{2}\right)
\end{array}\right]\left[\begin{array}{c}
x_{4} \\
\varphi
\end{array}\right] \\
& +\frac{2}{D_{1}}\left[\begin{array}{c}
B_{2} \\
1
\end{array}\right] i_{n}^{\prime} \\
i_{n}= & {\left[\begin{array}{ll}
0 & -\left(\alpha+\beta \varphi^{2}\right)
\end{array}\right]\left[\begin{array}{c}
x_{3} \\
\varphi
\end{array}\right] . }
\end{aligned}
$$

After calculating $i_{n}$, the reflected current is updated with

$$
i_{n}^{\prime \prime}=i_{n}^{\prime}+i_{n} .
$$

Similar to the Y-FD approach, the initial conditions for (26) are obtained from the HD representation of the system [5].

\section{B. Network of Transmission Lines}

In the case of a network with several transmission lines, the procedure described above could be used. The reflected currents are calculated for each line using an expression similar to (21). The solution for each load node can be calculated by using the nodal elimination as in (26). Finally, the reflected currents are updated.

\section{APPENDIX C \\ DATA FOR THE EXAMPLE}

The network used as example in Section IV is shown in Fig. 8. The network consists of 15 transmission lines and for simplicity of illustration, all of them have been taken with the same parameters and with a radial configuration. Each single-phase line is $100-\mathrm{km}$ long, with a height of $15 \mathrm{~m}$

$$
v_{2}=-\frac{\left(C_{2,1} x_{1}+C_{1,1} x_{2}+C_{1,2} x_{3}+C_{2,2} x_{4}+C_{1,3} x_{5}+C_{2,3} x_{6}+\alpha_{1} \varphi_{1}+\beta_{1} \varphi_{1}^{3}\right)}{\left(D_{1}+D_{2}+D_{3}\right)} .
$$




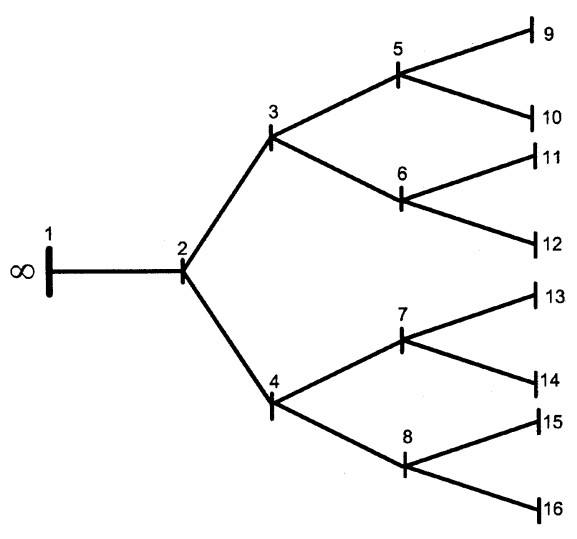

Fig. 8. Network corresponding to the example in Appendix A.

and conductor radius of $0.0158 \mathrm{~m}$. The load parameters are $\alpha=0.3$ for all nodes and $\beta=(2.7,4.3,4.3,5.5,5.5,5.5,5.5$, $6.1,6.1,6.1,6.1,6.1,6.1,6.1,6.1) \times 10^{-4}$. The generator at bus 1 is represented by a voltage source $v_{1}=15 \sin \left(\omega_{o} t\right)(\mathrm{kV})$, which corresponds to the input. The output corresponds to the source current. The perturbation applied to the system consists of the double exponential voltage $v_{\text {pert }}=70\left(e^{-t / T_{2}}-e^{-t / T_{1}}\right)(\mathrm{kV})$ with $T_{1}=150 \mu \mathrm{s}$ and $T_{2}=200 \mu \mathrm{s}$. The perturbation is applied at $t=3 \mathrm{~ms}$ with duration of $2 \mathrm{~ms}(\Delta t=1 \mu \mathrm{s})$.

\section{REFERENCES}

[1] A. Ramirez, A. Semlyen, and R. Iravani, "Order reduction of the dynamic model of a linear weakly periodic system-Part I: General methodology," IEEE Trans. Power Syst., vol. 19, pp. 857-865, May 2004.

[2] B. Gustavsen, "Computer code for rational approximation of frequency dependent admittance matrices," IEEE Trans. Power Delivery, vol. 17, pp. 1093-1098, Oct. 2002.

[3] N. Martins, L. T. G. Lima, and H. J. C. P. Pinto, "Computing dominant poles of power system transfer functions," IEEE Trans. Power Systems, vol. 11, pp. 162-170, Feb. 1996.
[4] B. Gustavsen and A. Semlyen, "Rational approximation of frequency domain responses by vector fitting," IEEE Trans. Power Delivery, vol. 14, pp. 1052-1061, July 1999.

[5] J. J. Rico, M. Madrigal, and E. Acha, "Dynamic harmonic evolution using the extended harmonic domain," IEEE Trans. Power Delivery, vol. 18, pp. 587-594, Apr. 2003.

[6] H. W. Dommel, Electromagnetic Transients Program Reference Manual (EMTP Theory Book). BC, Canada: Bonneville Power Administration, Dept. Elect. Eng., Univ. of British Columbia, Aug. 1986.

[7] J. K. Hale and S. M. V. Lunel, Introduction to Functional Differential Equations. New York: Springer-Verlag, 1993.

Abner Ramirez (M'96) received the B.Sc. from the University of Guanajuato Guanajuato, Mexico, in 1996, the M.Sc. degree from the University of Guadalajara, Guadalajara, Mexico, in 1998, and the Ph.D. degree from the Center for Research and Advanced Studies of Mexico (CINVESTAV), Campus Guadalajara, in 2001 .

He is currently a postdoctoral fellow in the Department of Electrical and Computer Engineering of the University of Toronto, Toronto, ON, Canada. His interests are electromagnetic transient analysis in power systems and numerical analysis of electromagnetic fields.

Adam Semlyen (LF'97) was born in 1923 in Rumania. He received the Dipl. Ing. degree from the Polytechnic Institute of Timisoara, Timisoara, Rumania, in 1950, and the Ph.D. degree from the Polytechnic Institute of Iasi, Iasi, Rumania, in 1965.

He began his career in Timisoara with an electric power utility and held academic positions at the Polytechnic Institute. In 1969, he joined the University of Toronto, Toronto, ON, Canada, where he is a Professor in the Department of Electrical and Computer Engineering (emeritus since 1988). His research interests include steady-state and dynamic analysis as well as computation of electromagnetic transients in power systems.

Reza Iravani (F'03) received the B.Sc. degree in electrcial engineering from Tehran Polytechnique University, Tehran, Iran, in 1976, and the M.Sc. and Ph.D. degrees in electrical engineering from the University of Manitoba, Winnipeg, MB, Canada, in 1981 and 1985, respectively.

He began his career as a Consulting Engineer. Presently, he is a Professor at the University of Toronto, Toronto, ON, Canada. His research interests include power electronics and power system dynamics and control. 